\title{
Rule- and idiosyncratically derived denominal verbs: Effects on language production and comprehension
}

\author{
MICHAEL H. KELLY \\ University of Pennsylvania, Philadelphia, Pennsylvania
}

\begin{abstract}
A distinction is drawn between two classes of denominal verbs, and four experiments examine the effects of this distinction on the production and comprehension of denominalizations. Rule-derived (RD) denominals are formed from nouns belonging to semantic categories whose members share the same meaning when they are used as verbs. For instance, denominal verbs formed from vehicles generally mean "to travel/convey by $x$," where $x$ represents the specific vehicle. In contrast, idiosyncratically derived (ID) denominals are drawn from categories whose members possess diverse meanings when they are used as verbs. Thus, to fish means "to try to catch fish," whereas to dog means "to chase tirelessly." Because the verb meanings of rule-derived terms are relatively predictable, they might be more easily produced and understood. Experiments 1 and 2 show that speakers are more likely to select RD terms for denominalization and are faster at creating denominal uses for RD terms. Experiments 3 and 4 show that RD denominals are rated as easier to understand than ID denominals, and that they are interpreted more uniformly across readers. The Discussion considers pragmatic accounts of the results, the theoretical basis for the distinction between RD and ID terms, and the more general point that experimental methods can be used to study creative uses of language.
\end{abstract}

When English speakers wish to denote a novel object, event, or concept, they have a variety of word formation devices at their disposal for meeting this vocabulary need. These devices include the affixing of derivational morphemes on an existing word (e.g., "work"+ "er"), combining two or more words into a compound word (e.g., "foot" +"ball"), and blending together portions of existing words (e.g., "smog" from "smoke" and "fog"; see Bauer, 1983, for a summary of English word formation). In this paper, I will examine one of these devices - namely, the extension of a noun into the verb category, which produces what is called a denominal verb. For example, bicycle and monkey originated in English as nouns but subsequently developed verb usages as well. Denominalization is a highly productive form of lexical innovation, and new examples are constantly entering English (e.g., e-mail). Nonetheless, despite the high productivity of denominal verb creation, not all nouns have assumed verb roles even though they are quite frequently encountered in English. Thus, even though we can say "I sledded to New Hampshire," "I jetted to New Hampshire," and "I biked to New Hampshire," we do not say "I carred to New Hampshire," despite the high frequency

This research was supported by NIH Grant 1 R29 HD23385, awarded to the author. Thanks to Kay Bock, Dorritt Billman, Liz Shipley, and two anonymous reviewers for helpful advice on this research, and to Caroline Fryd-Shechter and Cheryl Pfeiffer for technical assistance. Reprint requests may be addressed to the author at Department of Psychology, University of Pennsylvania, 3815 Walnut Street, Philadelphia, PA 19104-6196 (e-mail: kelly@cattell.psych.upenn.edu).

-Accepted by previous editor, Geoffrey R. Loftus of "car" as a noun. Such gaps in the lexicon of denominal verbs imply that restrictions exist on their creation and use. Clark and Clark (1979) have proposed one such restriction on denominal verb use derived from pragmatic considerations. This restriction, which Clark and Clark call the $d e-$ nominal verb convention, states that a noun can be used to convey meaning $X$ if the speaker has good reason to believe that the listener can readily infer meaning $X$, given their mutual knowledge.

This principle can account for the avoidance of many grammatical category extensions, including the example of car presented above. For instance, if people assume that a particular meaning will be represented by no more than one word, then a noun will not develop a verb use to convey meaning $X$ if an existing verb already represents meaning $X$. Thus, we do not use car as a verb meaning "to travel by car" because the verb drive already conveys exactly this meaning. Since the use of a vehicle word as a verb is generally interpreted as meaning "to travel by $\mathrm{X}$," where $\mathrm{X}$ stands for the vehicle, one cannot easily construct a meaning for She carred to Nome that would differ from She drove to Nome. Given a bias against the existence of true synonyms (Clark, 1983), the listener tries to find a way in which carred differs in meaning from drove, and, on not finding an alternative meaning readily available, judges carred to be used awkwardly.

Clark and Clark (1979) classify restrictions on words such as car under the heading "preemption by synonymy." However, this restriction is considered to be only one instantiation of the general denominal verb convention. Nonetheless, examples of this principle other than preemption by 
synonymy have not been explored. In this paper, I will examine another restriction on denominal verb formation derived from the denominal verb convention, one which involves nouns that are not blocked by existing verbs. The restriction to be discussed can be best introduced by examples. The following underlined denominal verbs were created by subjects in the experiments reported below: She streetcarred down to the wharf and Whenever he gets scared, he iguanas. Neither streetcar nor iguana has a verb listing in a recent edition of Webster's Ninth New Collegiate Dictionary (1986). Given the absence of established verb meanings for these words, the verb uses here must have been constructed rather than simply accessed from memory. I will argue that in trying to construct such meanings, English speakers can exploit a strategy for streetcar that is not available for iguana. In particular, a verb meaning for streetcar can be inferred through reference to other vehicle nouns that already have verb uses. Thus, examinations of dictionary entries reveal that vehicle terms share the same basic meaning when used as verbs: "To travel/convey by $X$," where $X$ represents the vehicle. By analogy, when streetcar is used as a novel verb, it probably means "to travel by streetcar," and this interpretation does seem to apply to the sentence above. The verb meaning for iguana, on the other hand, does not seem to be readily inferable from the verb meanings of other animal terms. For example, to dog means to chase tirelessly, to bird means to watch birds, and to pig means to eat sloppily and gluttonously. Thus, knowledge of the general category of animals does not seem to help very much in predicting what a particular animal term will mean when used as a verb. Rather, the meaning appears to depend on idiosyncratic aspects of the particular concept. Here, denominal verbs such as streetcar, whose meanings can be predicted from knowledge of the semantic categories from which the nouns were drawn, will be called rule-derived, abbreviated as $\mathrm{RD}$, whereas denominal verbs such as iguana, whose meanings depend on idiosyncratic aspects of their original nouns, will be called idiosyncratically derived, abbreviated as ID.

The purpose of this paper is to examine whether the distinction between RD and ID denominal verbs influences their production and comprehension. Given the relatively high predictability of RD meanings, they appear to satisfy more easily Clark and Clark's (1979) denominal verb convention than do ID meanings. In creating a denominal verb of the former sort, the speaker can have more confidence in the listener's ability to infer the meaning of the verb, given the assumption that the listener already knows some denominal verbs that follow the meaning rule for the particular category. Thus, with other things such as word frequency controlled, nouns would be predicted to develop verb uses to the extent that they come from a category whose nouns share similar meanings when they are used as verbs.

This hypothesis is difficult to test through historical records because "other things" were not controlled in the actual course of English history. For instance, when flavor- ings such as salt and pepper are used as verbs, they share the meaning "to add $X$ onto food," where $X$ stands for the flavoring. However, despite the rule-derived meanings of such denominal verbs, relatively few exist. This absence may be due to a variety of factors, such as the esoteric nature of the flavorings or the relative rarity of using words such as thyme and nutmeg compared with eye, shoulder, and other body parts that have idiosyncratic meanings when used as verbs. These problems with the historical record can be circumvented, however, by inspiring the creation of denominal verbs in the laboratory. In the first of four experiments, subjects were presented with pairs of nouns that lacked verb uses and were asked to use one of the nouns as a verb in a sentence. The nouns were controlled for word frequency, number of syllables, and stress pattern, but one of the nouns was drawn from a category whose denominal verbs shared similar meanings, whereas the other was drawn from a category whose nouns possessed idiosyncratic meanings. The denominal verb convention predicts that the former type of word should be more likely to be selected for verb use. A control condition was included in order to rule out the possibility that, for whatever reason, words from RD categories are in general easier to use than words from ID categories. In this control condition, subjects were presented with the same noun pairs and were simply instructed to use one word from each pair in a sentence. Since none of the nouns had existing verb uses, the subjects were expected to use the words in their standard noun senses. Since the rationale for the experiment was focused only on verb uses of the materials, no differences were expected between RD and ID categories when their instances were used as nouns.

\section{EXPERIMENT 1 Creating Denominal Verbs}

\section{Method}

Subjects. Thirty-six members of the University of Pennsylvania community were paid $\$ 4.00$ per hour for their voluntary participation in the 30- to 60-min experiment. In this and in other studies, all subjects were native speakers of English.

Materials. Six nouns from each of 12 categories were selected for the study, under the restriction that the nouns not have verb uses listed in Webster's Ninth New Collegiate Dictionary (1986). For six of the categories, nouns that develop verb uses tend to possess similar meanings, as determined by the Webster's dictionary. These categories were vehicles, colors, musical instruments, dances, flavorings, and human dwellings. The general meanings that denominal verbs from these categories possess are listed in Table 1 . Nouns from these categories will be called rule-derived (RD), since one can predict the meanings of denominal verbs in these cases from knowledge of the category to which they belong. Nouns from the remaining six categories possess relatively idiosyncratic meanings when they are used as verbs. These categories were animals, parts of the body, parts of buildings, tools, kitchen utensils, and articles of furniture. Some examples of existing denominal verbs from these categories, along with their varied meanings, are presented in Table 2. Nouns from these categories will be called idiosyncratically derived (ID), since one cannot easily predict the meanings of these denominal verbs from knowing only their superordinate category. 
Table 1

Rule-Derived Denominal Categories Used

in the Experiments, Along With General

Definitions of Verbs Formed From Each Category

\begin{tabular}{ll}
\hline \multicolumn{1}{c}{ Category } & \multicolumn{1}{c}{ Definition } \\
\hline Vehicle & To travel/convey by $X$ \\
Musical instrument & To play the $X$ \\
Dance & To dance the $X$ \\
Flavoring & To add $X$ to food \\
Color & To make something have the color of $X$ \\
Human dwelling & To occupy or live in $X$ \\
\hline
\end{tabular}

Each member of the RD categories was paired with one member from one of the ID categories, producing 36 pairs in all. Each RD category was thus paired exactly once with each ID category. The $\mathrm{RD}$ and ID items in each pair did not differ significantly in word frequency as determined by the Francis and Kučera (1982) frequency norms $[t(35)=0.20, p>.80]$. They also shared the same number of syllables and stress structure, and they were often similar phonolog ically (e.g., flamenco and flamingo) or morphologically (e.g., streetcar and backbone are both compound words). Word frequency was controlled to eliminate the possibility that subjects will simply select the more common word for denominalization. Stress and syllable number were controlled because English nouns and verbs differ systematically in these variables (see Kelly, 1992, for a review). For example, disyllabic nouns tend to have primary stress on the first syllable, whereas disyllabic verbs tend to have stress on the second syllable (Sherman, 1975). If speakers have abstracted these phonological differences, and transfer nouns to the verb class to the extent that they sound like typical verbs, then neglecting these variables could produce unanticipated biases in the subjects' choices. In fact, speakers are more likely to create denominal verbs from nouns that have the typical verb stress pattern (Kelly, 1988).

Despite close matching on many variables, the $R D$ and ID items did differ significantly in prototypicality within their semantic categories. Prototypicality was measured through the Battig and Montague (1969) category dominance norms, which have been shown to be highly correlated with prototypicality ratings (Mervis, Catlin, \& Rosch, 1976). In this norming study, 442 subjects were presented with the name of a category such as furniture and were asked to list as many instances of the category as they could in $30 \mathrm{sec}$. Whereas $\mathrm{RD}$ items were listed by a mean of 67 subjects, ID items were listed by a mean of only 33 subjects $[t(35)=2.59, p<.02]$. However, none of the experiments here produced significant correlations between the RD-ID prototypicality difference and performance on the denominal verb tasks. Indeed, as will be noted in the Results section of this and other studies, most of the correlations were slightly negative, meaning that greater typicality advantages for RD items were associated with smaller $\mathrm{RD}$ advantages on the experimental tasks. Hence it is unlikely that prototypicality influenced the results.

The noun pairs were randomly ordered and then printed in capital letters in two booklets, with six pairs on each page. In the first booklet, one member of each pair was printed directly above the other, with the order determined randomly, subject to the constraint that half the pairs list the RD item first and half list the ID item first. In addition, within each of the 12 categories, three items were listed above and three below their corresponding items from the other categories. Finally, the nominal categories to which the words belonged (e.g., vehicle) were printed above each pair in order to avoid possible ambiguities in interpretation, such as construing turquoise as a mineral rather than as a color. The category names were printed in a left to right order that matched the top-bottom order of their instances. Booklet 2 was identical to Booklet 1 except that the top-bottom arrangements were reversed. Each booklet was presented to 18 subjects.

Procedure. Eighteen subjects participated in a verb condition, and 18 in a no-verb condition. Half the subjects in each condition re- ceived Booklet 1, whereas the other half received Booklet 2. The subjects in the verb condition were told that the experimenters were interested in how meanings change when words are used as a new part of speech. Examples of denominal verbs were then provided, in order to illustrate grammatical category shifts. The subjects were then told that they would be asked to use nouns as verbs in sentences of their own invention. A series of 36 noun pairs were to be presented, and the subjects were instructed to select one member from each noun pair and use it as a verb in a sentence. A choice was justified on the grounds that the task was difficult (with which subjects indeed concurred). Thus, if they could not concoct a verb use for one of the nouns, perhaps they could for the other. Most subjects believed that their choices were of interest, but no one reported awareness of the rule versus idiosyncratic distinction prior to debriefing. Subjects in the no-verb condition received the same materials as did the subjects in the verb condition. However, these subjects were instructed to select one word from each pair and use it in a sentence, with the explanation that the experimenters were collecting normative data on word meanings. The subjects in this condition also believed that we were interested in their choices, but they were completely ignorant of the specific purpose of the study or its relation to denominal verb formation. Since none of the nouns used in the study possessed existing verb uses, no denominal verb innovations were expected in this condition, and none were found. Although the target words in this condition were used primarily as nouns, adjective uses also appeared, particularly for colors. However, the relevant predicted results for the two conditions were not altered by this fact.

\section{Results and Discussion}

The subjects in the verb condition were significantly more likely to create denominal verbs from $\mathrm{RD}$ rather than ID categories. The mean asymmetry was $59.0 \% \mathrm{RD}$ selection and $41.0 \%$ ID selection, a difference significant across both subjects $[t(17)=3.10, p<.01]$ and items $[t(35)=2.06$, $p<.05]$. Table 3 ranks each category in terms of the percentage of pairs for which its instances were selected for denominal verb use. It can be seen that the $\mathrm{RD}$ categories tend to be ranked higher than the ID categories, and a MannWhitney test indicated that the two types of categories were drawn from different populations $(U=4, p<.05)$.

This bias toward RD selection was restricted to denominal verb use. No differences between $R D$ and ID items were found in the no-verb condition [mean percentage of rule derived selection, 50.3 ; subjects $t(17)=0.17$; items $t(35)=$ 0.07 , both $p s>.85]$. Thus, the results in the verb condition cannot be attributed to the generally greater tendency

\section{Table 2}

Idiosyncratically Derived Denominal Categories Used in the Experiments Along With Selected Definitions

\begin{tabular}{ll}
\hline \multicolumn{1}{c}{ Category } & \multicolumn{1}{c}{ Definition } \\
\hline Animal & $\begin{array}{l}\text { To attempt to catch fish ("fish") } \\
\text { To act in a mischievous manner ("monkey") } \\
\text { To bear without overt reaction ("stomach") } \\
\text { To look at ("eye") }\end{array}$ \\
Building part & To surround with a wall ("wall") \\
Kitchen utensil & $\begin{array}{l}\text { To unite or make firm ("cement") } \\
\text { To take up in a spoon ("spoon") }\end{array}$ \\
Tool & To cover with a lid ("lid") \\
& To strike blows ("hammer") \\
Furniture & To fasten with a nail ("nail") \\
& To install in office ("chair") \\
& To remove from consideration ("table")
\end{tabular}


Table 3

Mean Percentage of Nouns Selected for Verb Use From the Rule-Derived (RD) and Idiosyncratically Derived (ID) Categories in Experiment 1

\begin{tabular}{lll}
\hline \multicolumn{1}{c}{ Category } & Rule & $\%$ \\
\hline Vehicle & RD & 67 \\
Musical instrument & RD & 66 \\
Flavoring & RD & 63 \\
Dance & RD & 62 \\
Kitchen utensil & ID & 59 \\
Building part & ID & 57 \\
Dwelling & RD & 47 \\
Color & RD & 43 \\
Tool & ID & 41 \\
Furniture & ID & 34 \\
Animal & ID & 32 \\
Body part & ID & 23 \\
\hline
\end{tabular}

to use terms from RD categories in sentences. Rather, differences between RD and ID categories were restricted to verb use, as predicted. The results also cannot be attributed to the greater typicality of RD items. The typicality difference between the RD and ID members of each pair correlated only -.06 and -.14 with the percentage of subjects who selected the RD item for use in the verb and no-verb conditions, respectively (both $p s>.40$ ). Indeed, the slight negative correlations mean that typicality, if anything, inhibited rather than facilitated word selection.

Although the results are consistent with the category predictor bias, further confidence would be gained if it could be shown that the results were indeed related to the ease of predicting the verb meanings of RD terms. One could examine this in more detail by exploiting differences among the individual items in the extent to which they were used as verbs. Thus, whereas elevator was selected for verb use by $89 \%$ of the subjects, tractor was selected by only $39 \%$ of the subjects. Part of the difference probably derives from the fact that they were paired with different terms. Whereas elevator was paired with alligator, tractor was paired with skillet. It may be easier to think of a verb use for skillet than for alligator, and hence the selection of tractor might drop relative to elevator. However, the difference might also depend on the extent to which these words follow their category rule for denominal verbs. If tractor is more likely than elevator to violate the "travel/convey by $X$ " rule for denominal verbs, it might be selected less often. In fact, tractor sometimes did possess an idiosyncratic meaning when used as a verb, as illustrated by the sentence "Joe tractored the entire field and got his work done." This sentence clearly does not mean "Joe traveled to the entire field by way of tractor," but rather something like "Joe plowed the entire field using a tractor." In general, if the rule-idiosyncratic distinction partially underlies the results of this experiment, one would expect to find a significant correlation between the extent to which a particular RD noun was used as a verb and the extent to which such uses followed the category rule.

In order to test this hypothesis, the sentences with terms from $\mathrm{RD}$ categories were presented to a rater naive to the purposes of the study. The rater was given the list of meanings shown in Table 1 and was asked to classify each sentence as following or violating the rule for the relevant category. No further instructions or examples were given, but the rater found the procedure fairly straightforward. The percentage of sentences that followed the category rule was calculated for each item. These percentages were then correlated with the percentage of subjects who selected each RD term for use as a denominal verb. The significant .58 Pearson correlation $(p<.001)$ indicates that nouns are made into verbs to the extent that they regularly evoke the meaning rule for their category. Thus, the distinction between RD and ID denominal verbs applies not only to categories as a whole, such as vehicles versus animals, but seems to operate within categories as well.

In sum, the distinction between RD and ID denominal verbs appears to affect the speaker's selection of which nouns to extend to the verb class. However, one could argue that the proposed distinction is not clear-cut. For example, I classified vehicles as RD denominals since they all mean "to travel or convey by $X$," where $X$ is the vehicle. On the other hand, animal terms seem to possess very diverse meanings when used as verbs. Thus, whereas monkey means "to clown around," dog means "to chase tirelessly." However, one might claim that animal denominal verbs are $R D$ as well, since they all seem to mean "to behave like $\mathrm{X}$," where $\mathrm{X}$ is the animal. I will present three responses to this criticism.

First, one can argue that certain subareas of idiosyncratic categories group together in systematic ways, though in comparison with "pure" RD categories, they contain many exceptions. Thus, to fish does not mean "to act like a fish" but rather to catch fish. To bird does not mean "to act like a bird," but rather to watch birds. Now, it must be admitted that RD and ID denominals are not distinctly discrete. Rather, they appear to fall along a continuous dimension. Nonetheless, the dimension might be relevant to the creation of denominal verbs, and the best strategy to demonstrate this relevance would focus on the extreme ends of the continuum. The categories used in this experiment seem to be located toward the two extremes, and the results support the intuitive division.

Second, one could point to the pragmatics of lexicography to demonstrate the relative homogeneity of $R D$ denominals. Lexicographers clearly follow pragmatic rules in writing definitions. Rather than include every component of a word's meaning in a definition, they assume that the reader will be able to make some easy inferences, such as knowing that being a bird entails being an animal. Since lexicographers will assume that readers possess such knowledge, they will not include an explicit animal entry under bird definitions. Lexicographers therefore appear to follow Gricean maxims of communicative efficiency (Grice, 1975). A definition should include just enough information for the reader to gain a reasonable understanding of a word's meaning. If this pragmatic rule is kept in mind as one examines actual definitions of denominal verbs, one will find that almost identical definitions are provided for 
RD denominals, whereas strikingly different definitions are given for ID denominals. Thus, actual definitions of vehicle verbs state "To travel by $X$," with the $X$ replaced by the specific vehicle being discussed. Lexicographers apparently assume that no more information is required for the reader to infer the meaning of vehicles whose names are used as verbs. Now, if animal verbs such as monkey, $d o g$, or pig are consulted, one does not find a general definition "To behave like an $X$." Rather, much more specific definitions are provided, which differ from animal to animal. One can conclude, therefore, that lexicographers believe that more idiosyncratic definitions are needed for one to understand such verbs.

Finally, one can construct and test further hypotheses that might be expected to follow from the rule/idiosyncratic distinction, the goal being to obtain converging evidence for the division. For example, rather than giving English speakers the freedom of choosing which nouns to use as verbs, as in the first experiment, suppose that we force subjects to invent a sentence in which they use a particular noun as a verb. If subjects are timed while they try to come up with a verb meaning for various nouns, one would expect RD nouns to require less time for invention than ID nouns do. The rationale is that one can very easily formulate a meaning for $\mathrm{RD}$ terms by means of analogy with existing denominal verbs drawn from the same nominal category. Such a strategy would be unavailable for ID nouns. This hypothesis was tested in Experiment 2. As in Experiment 1, a control condition was included in which subjects were asked to use each word in a sentence with no reference to a verb use. Since none of the nouns possessed an established verb use, no such uses were anticipated in this condition. In this case, the times needed for creating sentences containing RD terms should not differ from the times needed for creating sentences containing ID terms.

\section{EXPERIMENT 2 Timing Denominal Verb Creation}

\section{Method}

Subjects. Forty-eight members of the University of Pennsylvania community were paid $\$ 4.00$ per hour for their voluntary participation in this 30 - to 45 -min study.

Materials. The 72 words used in Experiment 1 were retained here and divided into two lists of 36 words. The first list was created by randomly selecting three nouns from each of the six RD categories along with the terms from the ID categories with which they were yoked. The remaining 36 words constituted the second list. The words from the same category were blocked in each list, thus forming 12 blocks of 3 words. Blocking items by category may help subjects to access the rule governing each RD category. One might therefore expect that sentence generation times for RD terms should decrease within a block. Blocking terms from the same ID category should provide little benefit, since the meanings of denominal verbs in these cases depend more on the particular term than on the superordinate category.

The categories in each list were arranged in two orders. The first order was determined randomly, subject to the constraint that $\mathrm{RD}$ and ID categories alternate. The second order was obtained by reversing the first. The same category orders were used in both lists. All 6 orders of items within each category were also used, creating a grand total of 12 orders for each list ( 2 between-category orders $x$ 6 within-category orders).

Procedure. Twenty-four subjects participated in a verb condition and 24 participated in a no-verb condition. Half the subjects in each condition received List 1 , whereas the other half received List 2. Each of the 12 orders for the two lists was thus presented to 1 subject within the verb condition and 1 subject within the no-verb condition. In addition, each noun was presented to 24 subjects, half in the verb condition and half in the no-verb condition.

The subjects in the verb condition were told that they would see a series of nouns on a Macintosh Plus computer screen. Prior to each word presentation, the subject was asked to fixate a central cross. After $3 \mathrm{sec}$, one of the 36 nouns appeared on the screen. The subject was asked to create a sentence in which the noun was used as a verb. After thinking of a sentence, the subject pressed the space bar on the computer keyboard, which prompted the computer to store the time between the onset of word presentation and the space bar response. After pressing the space bar, the subject wrote the sentence on note paper. The subject then pressed the space bar once again, which led to the reappearance of the fixation cross for the 3-sec preparation period. The subjects in the no-verb condition received exactly the same procedure, except that they were asked to invent any sentence containing the target word, with no mention of a verb use.

\section{Results and Discussion}

Two analyses of variance were performed on the generation time data, one with subjects as the random factor and one with items as the random factor. Both analyses contained the three factors of task (verb or no verb), word class (RD or ID), and trial within a block (first, second, or third). Each subject received both RD and ID terms, but used them either as verbs or not, depending on instructions. Thus, in the subjects analysis, word class was a withinsubjects factor and task was a between-subjects factor. Each of the nouns appeared in both verb and no-verb conditions, so task was a within-items factor in the items analysis. Since the nouns were presented individually in this study rather than in explicit pairs, as in Experiment 1, word class was treated as a between-items factor in the items analysis. For both the subjects and items analyses, the trial factor was a within variable.

The analyses revealed that using nouns in their standard senses required less time than did creating denominal verbs [ 2.52 vs. $8.31 \mathrm{sec}$; subjects, $F(1,46)=15.55$; items, $F(1,70)=224.66$; both $p s<.001]$. In addition, inventing sentences with $\mathrm{RD}$ words required less time than inventing sentences with ID words [4.88 vs. $5.95 \mathrm{sec}$; subjects, $F(1,46)=10.05$; items, $F(1,70)=7.51$; both $p$ s $<.01$ ] However, these main effects were qualified by a significant interaction between type of task and word class [subjects, $F(1,46)=9.54$; items, $F(1,70)=8.07$; both $p$ s $<.01$ ] As Table 4 shows, the significant interaction derives from a difference between RD and ID words in the verb condition that is absent in the no-verb condition. Simple effects comparisons verified that RD words were used more rapidly as denominal verbs than were ID words [subjects, $F(1,46)=19.58$; items, $F(1,70)=8.02$; both $p s<.01]$. However, no differences between the word classes emerged when they were used as nouns or adjectives in the no-verb condition [subjects, $F(1,46)=0.03$; items, $F(1,70)=0.02$ ]. As in Experiment 1, the RD advantage found in the verb 
Table 4

Mean Times (in Seconds) to

Create Sentences in Experiment 2

\begin{tabular}{ccc}
\hline & \multicolumn{2}{c}{ Condition } \\
\cline { 2 - 3 } Category & Verb & No Verb \\
\hline RD & 7.23 & 2.51 \\
ID & 9.40 & 2.54
\end{tabular}

Note- $R D$, rule-derived; ID, idiosyncratically derived.

condition cannot be attributed to the greater typicality of RD items. In particular, the greater the category dominance of the RD member of an RD-ID pair, the less was its speed dominance as measured by subtracting the generally faster RD time from the ID time. This pattern appeared in both the verb $(r=-.15)$ and no-verb $(r=-.06)$ conditions, though neither of these correlations was significant (both $p s>.35$ ).

As one would expect, creating denominal verbs requires more time than using words in their standard grammatical class, regardless of the rule versus idiosyncratic distinction (all $F \mathrm{~s}>9.94$; all $p s<.005$ ). The only other significant effect obtained was that of the trial factor in the subjects analysis $[F(2,92)=3.25, p<.05]$. This effect was due to faster sentence formation times in the second of the three trials within a block than in the third $[t(47)=2.29, p<$ $.05]$. I have no explanation for this finding; but it was not replicated in the items analysis $[F(2,140)=1.70, p>.15]$, and it did not interact significantly with any of the other factors in the experiment. Placing RD category members together clearly did not facilitate denominal formation times for later trials in a block. However, it is difficult at this point to make strong conclusions about the absence of a blocking effect, given the current lack of a detailed processing model for denominal verb production.

In Table 5, the categories are ranked according to the mean time that it took to generate sentences in which their instances were used as denominal verbs. Though the $R D$ categories tended to cluster at the fast end of the scale, a Mann-Whitney test did not support a statistical distinction between the RD and ID classes $(U=9, p>.10)$. However, the results found using the generation time measure parallel

Table 5

Rule-Derived (RD) and Idiosyncratically

Derived (ID) Categories Ranked According to Mean Time (in Seconds) Needed

for Creating Denominal Verb Sentences

\begin{tabular}{lll}
\hline \multicolumn{1}{c}{ Category } & Rule & Time \\
\hline Vehicle & RD & 5.30 \\
Dance & RD & 5.71 \\
Kitchen utensil & ID & 6.57 \\
Flavoring & RD & 6.73 \\
Dwelling & RD & 7.10 \\
Tool & ID & 7.75 \\
Building part & ID & 7.97 \\
Musical instrument & RD & 8.46 \\
Animal & ID & 9.55 \\
Color & RD & 10.02 \\
Furniture & ID & 10.28 \\
Body part & ID & 14.29 \\
\hline
\end{tabular}

closely the patterns found in Experiment 1 using the choice measure. Thus, the ranks of the categories in Table 5 are highly correlated with the ranks in Table 3 (Spearman $\rho=$ $.76, p<.02$ ), indicating that the categories which most quickly inspire denominal verb uses also tend to be selected for denominalization.

As with Experiment 1, each RD denominal verb sentence generated by the subjects was classified by a rater as following or violating the rule for its category. The rater was not the one who participated in the first experiment, but received the same instructions. For each item, the percentage of sentences that followed their relevant rule was calculated. These percentages were then correlated with the percentage of subjects who chose each RD item for denominalization in Experiment 1 and with the mean sentence creation times in Experiment 2. Items that followed their denominalization rule more strictly were also more likely to be denominalized $(r=.33, p<.05)$. However, no significant relation was found between rule conformity and time needed to create a denominalization $(r=-.09)$, although the trend was for greater rule conformity related to faster generation times, as would be predicted. One reason for the absence of strong relations between rule conformity and other measures of denominal production could be the generally high levels of rule conformity obtained. Seventy-two percent of all the RD denominal sentences were rated as following the rule for their categories. In addition, for 23 of the $36 \mathrm{RD}$ items, at least $75 \%$ of the sentences were classified as agreeing with their rule. Such high levels of rule conformity would be expected, given that the most extreme cases of RD categories were selected for these experiments. That some significant relations between rule conformity and denominalization were still obtained indicates that the category predictor bias is an important principle in denominal verb formation.

In sum, the first two experiments support a distinction between RD and ID denominal verbs in language production. However, the distinction has not as yet been shown to operate in language comprehension. Perhaps RD nouns are selected for verb use because speakers find it easier to construct verb meanings for such terms. However, once constructed, verb meanings for ID terms might be just as easy to understand. If this is the case, then attributing the selection patterns found here to pragmatic principles such as the category predictor bias or, more generally, the denominal verb convention would be called into question. The distinction between $\mathrm{RD}$ and ID denominal verbs might be relevant to language production processes, but would have few consequences for language comprehension. The next two experiments were performed to examine whether RD denominal verbs are easier to understand than ID denominal verbs.

\section{EXPERIMENT 3 Judgments of Comprehension Ease}

In this experiment, subjects were presented with pairs of sentences, with one sentence in each pair containing an 
Table 6

Mean Percentage of Rule-Derived (RD) and Idiosyncratically Derived (ID) Sentences Judged Easier to Comprehend in the Verb and No-Verb Conditions in Experiment 3

\begin{tabular}{lccc} 
& \multicolumn{3}{c}{ Judgment Type } \\
\cline { 2 - 4 } Condition & RD Easier & ID Easier & No Difference \\
\hline Verb & 53 & 18 & 29 \\
No-verb & 32 & 26 & 42 \\
\hline
\end{tabular}

RD denominal verb and the other sentence containing an ID denominal. The subjects were asked to read each pair and to place a check next to the sentence that seemed easier to understand. If the sentences seemed equally comprehensible, they could check the phrase "no difference." If the rule/idiosyncratic distinction is relevant to comprehending denominal verbs, sentences containing RD denominals should be rated easier to understand than sentences containing ID denominals. As with the production studies, this comprehension experiment included a control condition in which the target word pairs appeared in sentences as nouns or adjectives. The subjects in the control condition were also asked to judge which, if any, member of a pair was easier to understand. No differences between RD and ID terms were expected in this condition.

The rule and idiosyncratic pairs from the prior experiments were also used here. Rather than creating new sentences for this study, which might contain inadvertent experimenter biases, I randomly selected sentences invented by the subjects in Experiment 2 for comprehension judgments. Sentences from Experiment 1 were less desirable, because subjects invented sentences for only one member of each RD-ID pair. The comprehension hypotheses would then have to be tested through sentences created by different speakers. In addition, since subjects wrote more sentences for RD denominal verbs than for ID denominal verbs, they had more practice on the average with RD items, which might lead to more understandable sentences for these cases. In contrast, subjects in Experiment 2 used both members of an RD-ID pair as a denominal verb and created the same number of ID-based sentences as RD-based sentences. Hence, materials for the comprehension experiment were drawn from Experiment 2 sentences.

\section{Method}

Subjects. Forty members of the University of Pennsylvania community were paid $\$ 4.00$ per hour for their voluntary participation in this 20 - to 30 -min study.

Materials. Two lists of 72 sentence pairs were created, one with the noun pairs from Experiment 1 as denominal verbs, and the other with them as nouns or, in a few cases, adjectives. The first list corresponded with a verb condition; the second, with a no-verb condition. Sentence pairs were randomly selected from those created in Experiment 2, with the constraints that (1) both sentences in a pair were created by the same speaker, (2) each of the Experiment 2 subjects was represented three times, and (3) each noun pair was represented four times, twice in the verb and twice in the no-verb condition The sentence pairs in each condition were arranged randomly on computer-printed sheets, with the constraints that (1) at least 35 sentence pairs separate the two presentations of the same target pair,
(2) the sentence containing the RD term be placed above the corresponding sentence containing the ID term for half the pairs, and vice versa for the remaining half, and (3) for the two pairs containing the same target words, one pair listed the RD items above the ID item, whereas the other pair had the opposite ordering. A dash was printed before each sentence and also before the phrase "no difference," which was printed below the sentences in each pair. The subjects wrote their comprehension judgments on these dashes. A randomly selected sample of sentence pairs from the verb and no-verb conditions is listed in Appendix B.

Procedure. Twenty subjects participated in the verb condition and 20 participated in the no-verb condition. The subjects in both conditions were told that they would read 72 pairs of sentences. They were asked to place a check in the dash next to the sentence in each pair that seemed easier to understand. If the sentences seemed equally easy or difficult to understand, the subjects were instructed to place a check next to the phrase "no difference." Though no other details were given about what was meant by "ease of understanding," the subjects felt that the task was straightforward, and the procedure did produce systematic, interpretable results.

\section{Results and Discussion}

Table 6 shows the percentage of RD, ID, and nodifference judgments made by the subjects in the verb and no-verb conditions. A very large difference was obtained between the RD and ID terms when they were used as verbs, with sentences containing RD denominal verbs rated as being much easier to understand [subjects, $t(19)=12.31$; items, $t(35)=5.18$; both $p s<.01] .{ }^{1}$ However, in the no-verb condition, sentences with RD terms were also rated as being easier to understand than sentences with ID terms. Though small, this difference was significant across subjects $[t(19)=3.31, p<.01]$, but not across items $[t(35)=$ $1.11, p \geq=.20$ ]. Although I have no explanation for the effects obtained in the no-verb condition, the strength of the difference between the RD and ID terms is more than five times larger in the verb condition than in the no-verb condition, which is statistically significant [subjects, $t(38)=$ 8.75 ; items, $t(35)=3.00$; both $p s<.01$ ]. Also, the no-verb condition elicited more "no difference" judgments than did the verb condition [subjects, $t(38)=1.91, p<.07$; items, $t(35)=4.46, p<.0001]$, which further supports the conclusion that RD and ID items show comprehension differences primarily when they are used as denominal verbs rather than in their standard grammatical roles.

As in the prior experiments, the results cannot be attributed to the greater typicality of RD items. The difference in typicality between the RD and ID members of a pair correlated only -.15 and .18 with their difference in rated comprehension ease in the verb and no-verb conditions, respectively. Neither correlation is significant (both $p \mathrm{~s} \geq$ .25 ), and the pattern in the verb condition is in the opposite direction from what would be predicted by the hypothesis that the denominal verb advantage for $\mathrm{RD}$ items is due to their typicality advantage.

Table 7 lists the rule and idiosyncratic categories ranked according to the strength with which their instances were judged easier to understand when used as verbs. One can see that the RD and ID categories are almost completely segregated. Denominal verbs of the former sort are overwhelmingly rated as easier to comprehend. A Mann- 
Table 7

Rule-Derived (RD) and Idiosyncratically Derived (ID) Categories Ranked in Terms of Mean Percentage of Denominal Verb Uses Judged Easier to Understand

\begin{tabular}{lcc}
\hline \multicolumn{1}{c}{ Category } & Rule & $\%$ \\
\hline Flavoring & RD & 70.0 \\
Vehicle & RD & 61.3 \\
Musical instrument & RD & 61.2 \\
Dance & RD & 61.2 \\
Dwelling & RD & 40.2 \\
Tool & ID & 29.3 \\
Color & RD & 26.8 \\
Kitchen utensil & ID & 25.0 \\
Furniture & ID & 16.0 \\
Building part & ID & 15.3 \\
Animal & ID & 14.3 \\
Body part & ID & 9.8 \\
\hline
\end{tabular}

Whitney test indicates that the RD and ID categories are drawn from different populations $(U=1, p<.01)$. The rank order of the categories in the comprehension judgment study correlates highly with the ranks in the choice study (Spearman $\rho=.84, p<.01$ ) and the generation time study (Spearman $\rho=.70, p<.05$ ), suggesting a close relationship between the ease with which different nouns can become denominal verbs and the ease with which such uses are understood.

\section{EXPERIMENT 4 Paraphrase Agreement}

The measure of comprehension used in Experiment 3 did not provide information about the interpretations that the subjects gave to the various sentences. The final experiment was designed to gather such information, and to test another comprehension consequence of the rule-idiosyncratic distinction. In particular, if the meanings of RD denominal verbs are more transparent than the meanings of ID denominal verbs, readers should agree fairly well on interpretations of sentences containing $R D$ denominals. With sentences containing ID denominals, on the other hand, readers should show more variability in their interpretations. Such variability would suggest that speakers would not be able to convey their intended meanings as easily using ID denominals as they could using RD denominals.

The subjects in this experiment were presented with a subset of the sentences used in the verb condition of Experiment 3 . They were asked to write a paraphrase for each sentence, indicating the meaning of the denominal verb. Across the paraphrases generated for each denominal verb, the ratio of word types to word tokens was then calculated, which provided a measure of semantic overlap among the set of paraphrases. The assumption underlying the use of this measure was that sentences interpreted in a similar manner by different readers should be paraphrased with many of the same words. If RD denominal verbs are less ambiguous than ID verbs, the paraphrases should share more of the same vocabulary. Hence, the ratio of word types to word tokens should be lower for $\mathrm{RD}$ than for ID denominals.

A corresponding no-verb condition was not included, because the instruction to paraphrase a sentence that was already easily interpretable seemed strange and awkward. How, for example, does one paraphrase "The hamster ate the lettuce" so that the meaning of the original sentence is better expressed? Given that the first three experiments produced consistent effects of the rule-idiosyncratic distinction that were absent or smaller in the no-verb conditions, the inclusion of a no-verb condition in the paraphrase study was judged to be unnecessary.

\section{Method}

Subjects. Twelve members of the University of Pennsylvania community were paid $\$ 4.00$ per hour for their voluntary participation in this 60- to 70-min experiment.

Materials. Thirty-six sentences were randomly selected from the verb condition of Experiment 3, with the constraint that each RD and ID term be used exactly once. These sentences were then randomly printed on computer sheets, with no more than three RD or ID sentences appearing consecutively.

Procedure. The subjects were given examples of denominal verbs and potential paraphrases of them. None of these examples was drawn from the categories used in the experiment. The subjects were then told that they would read a series of sentences containing a denominal verb. They were asked to decide what the speaker might have meant by each sentence, and then to write a paraphrase of this meaning on an answer sheet provided by the experimenter.

For the 12 paraphrases given to each denominal verb, the number of word types and word tokens was tallied. The number of word tokens was determined by simply counting the number of words used in all 12 paraphrases for each denominal verb. For word tokens to be classified as members of the same type, they had to be spelled identically. Thus, different tense versions of verbs were classified as different types, as were plural and singular versions of nouns. One might question this scoring system, since word tokens with highly similar meanings were classified separately. However, the system was conservative and objective, in that no criterion besides total identity was used to classify two words as tokens of the same type. Furthermore, variations in the tense of a verb or the number of a noun have different semantic entailments. Since the experiment was concerned with semantic interpretations of denominal verbs, this scoring criterion seemed most acceptable (or least unacceptable). A complete set of paraphrases and corresponding type/token ratios are shown in Table 8 for an RD and ID denominal.

\section{Results and Discussion}

If the 12 paraphrases of a particular denominal verb had no words in common, the number of types would equal the number of tokens, and so the ratio of types to tokens would be 1 . As the overlap in vocabulary increases, the ratio of types to tokens drops. The mean type/token ratio for paraphrases of RD denominals was .22 , whereas the mean ratio for paraphrases of ID denominals was .25. Although small, this difference was significant $[t(70)=2.01$, $p<.05$ ], and it indicates more vocabulary overlap among paraphrases of $\mathrm{RD}$ denominals than among paraphrases of ID denominals. If vocabulary overlap reflects semantic overlap, these results indicate that $\mathrm{RD}$ denominals are interpreted more uniformly than ID denominals. As in the 
prior studies, the results are not due to the greater typicality of RD words. The typicality advantage of the RD word in an RD-ID pair correlated only .21 with the difference between their type/token ratios $(p>.20)$.

Table 9 ranks the 12 categories from smallest to largest type/token ratio. Although the RD categories tend to be clustered at the small end of the scale, a Mann-Whitney test did not indicate that the RD and ID categories were drawn from different populations $(U=10, p>.10)$. In addition, although the rankings in this study were correlated positively with the rankings from the three prior studies (paraphrase vs. choice production, .69; vs. generation time, .40; vs. comprehension judgments, .35 ), they were only significantly correlated with the choice rankings from Experiment $1(p<.01)$. The relative weakness of the effects in this study may derive partially from the fact that only a single denominal verb use of each noun was presented for paraphrasing, and these selections were not screened for whether they followed the verb rule of their category. Thus, the sentence for tractor was "He tractored the farm." Tractor in this case clearly does not mean "He traveled to the farm via tractor," but instead means something such as "He performed the farm work using the tractor." Violations of category rules may have obscured to some extent the difference between RD and ID interpretability. ${ }^{2}$ In fact, the tractor sentence was generally judged less easy to understand than its ID counterpart in Experiment 3 ("He cellared the boxes") and had a higher

Table 8

Set of Paraphrases and Type/Token Ratios for a Rule-Derived (RD) and an Idiosyncratically Derived (ID) Sentence Used in Experiment 4

\begin{tabular}{l} 
RD Sentence: I harmonicaed by the campfire. \\
Paraphrases: (Type/token $=.15$ ) \\
He played the harmonica by the campfire \\
I played my harmonica by the campfire \\
I played my harmonica by the campfire \\
I played the harmonica \\
I played the harmonica by the campfire \\
I played the harmonica by the campfire \\
I played the harmonica by the campfire \\
I played the harmonica by the campfire \\
I played the harmonica by the campfire \\
I played the harmonica by the campfire \\
I put out the campfire \\
Played the harmonica \\
ID Sentence: She balconied over to Romeo. \\
Paraphrases: (Type/token = .34) \\
She bent over the balcony to Romeo \\
She climbed over the balcony to get to Romeo \\
She hastened towards Romeo \\
She jumped from balcony to balcony \\
She leaned over the balcony towards Romeo \\
She made her way over to Romeo on the balcony \\
She met Romeo at the balcony \\
She moved across the balcony to Romeo \\
She spoke from the balcony to Romeo \\
She tiptoed (as if walking on a balcony) over to Romeo \\
She walked across the balcony to Romeo \\
She walked across the balcony to talk with Romeo \\
\hline
\end{tabular}

Table 9

Rule-Derived (RD) and Idiosyneratically Derived (ID) Categories Ranked According to Type/Token Ratio

\begin{tabular}{lll}
\multicolumn{1}{c}{ Category } & Rule & Ratio \\
\hline Musical instrument & RD & .173 \\
Vehicle & RD & .192 \\
Kitchen utensil & ID & .200 \\
Color & RD & .217 \\
Building part & ID & .232 \\
Animal & ID & .235 \\
Dance & RD & .242 \\
Flavoring & RD & .245 \\
Dwelling & RD & .247 \\
Tool & ID & .250 \\
Furniture & ID & .287 \\
Body part & ID & .297 \\
\hline
\end{tabular}

type/token ratio in this experiment (.20 for tractor vs. .15 for cellar). Despite these problems, the significant relationship between the choice and paraphrase results indicates that the nouns selected most often for denominalization were also interpreted more uniformly by readers. ${ }^{3}$

In addition to testing the primary hypothesis of Experiment 4, the paraphrases provide the opportunity for examining a possible confound that might have created an inadvertent bias in favor of RD items. Suppose that the ID nouns were more difficult to denominalize because their verb meanings were more likely than $R D$ verb meanings to be synonymous with existing verbs. The bias against synonymy described in the introduction would then block ID denominals more than RD denominals. The paraphrases from this experiment were examined to check this possibility. In particular, suppose that someone used car as a denominal verb in the sentence "John carred to New York." A reasonable paraphrase is "John drove to New York," in which a single existing verb replaces the original denominal verb. In contrast, the modal interpretation of the sentence "She balconied over to Romeo" in Table 8 is "she walked/moved across the balcony to Romeo." In this case, balcony was replaced by an entire phrase, because there is no single verb in English that is synonymous with the denominal meaning "to move or walk across a balcony." I therefore examined the paraphrases of Experiment 4 to see whether the denominal verbs were translated into phrases or single existing verbs, with the latter implying a potential hindrance to denominalization. In all cases except one, the denominal verbs were translated into phrases. Thus, the modal translation of casserole was "put into a casserole" and the modal translation of harmonica was "played the harmonica." Only esophagus was replaced with a single verb: swallowed. So, it is unlikely that this synonymy factor played a significant role in the RD denominalization advantages.

\section{GENERAL DISCUSSION}

According to Clark and Clark's (1979) "denominal verb convention," a noun can be used as a verb if the speaker believes that the listener can readily infer the meaning of 
the denominal verb, given their mutual knowledge. In order to test the validity of this convention, a variable must be found that affects the speaker's selection of denominal verbs, while at the same time predicting the ease with which different denominal verbs are understood. The particular variable examined here, the distinction between RD and ID denominal verbs, appears to satisfy these two criteria. First, speakers are more likely to create denominal verbs from RD rather than ID nouns, and they are faster in denominalizing the former nouns. Second, RD verbs are rated as easier to comprehend than ID verbs, and they are interpreted more uniformly than ID verbs. The comprehension results indicate that speakers have less justification to assume that listeners can interpret ID verbs as easily as $R D$ verbs. In addition, the coupling between the production and comprehension results is remarkably tight. This coupling can be illustrated by comparing the results of Experiments 1 and 3. Both experiments involve making a choice between RD and ID items, but differ in that Experiment 1 subjects had to choose nouns for denominalization, whereas Experiment 3 subjects had to choose the denominal verb sentences that were most easily understood. I have already noted that the categories selected most often for denominalization in language production are also the ones whose members are judged to be most easily understood when denominalized. This correlation between production and comprehension extends down to individual terms. Thus, the extent to which various nouns were denominalized in Experiment 1 correlated .64 with the extent to which they were rated as the more understandable member of their pairs in Experiment $3(p<.01)$.

Despite the close correspondence between the production and comprehension data, it is still not clear that $R D$ nouns are chosen for denominalization because of speaker beliefs about the listener. RD nouns might be denominalized for the simple reason that speakers can invent verb meanings for these terms more easily than for ID terms. On the receiver side, $\mathrm{RD}$ denominals would be more readily understood for the same reason that they are selected for denominalization: Their meanings follow predictable rules based on their nominal category.

The pattern of results that would seem most congruent with pragmatic accounts would have replicated the two comprehension experiments and the first production experiment, but would have failed to show any differences between RD and ID nouns in the time taken to create a denominalization. Such a pattern would indicate that RD nouns were not easier than ID nouns to denominalize, but were still more likely to be selected for denominalization. The most parsimonious explanation for such results would attribute speaker selection biases to implicit concern for listener comprehension ease.

An explanation based solely on production difficulty would have predicted biases toward selecting RD nouns for denominalization, with no differences in rated comprehension ease or uniformity of interpretation. Rather, verb uses for ID nouns would simply be more difficult to invent than verb uses for RD nouns, but, once invented, would be just as easy to comprehend. This interpretation would be further supported if ID denominalizations took more time to invent. The significant comprehension effects that were found, however, are consistent with at least a partial pragmatic account of the production biases.

The difficulty of evaluating pragmatic interpretations of speaker behavior appears in other areas besides the denominalization phenomena discussed here. For example, Fowler and Housum (1987) have shown that the second mention of a word denoting a particular object tends to be less stressed than the first mention. In more functionalist terminology, words denoting given information tend to be less stressed than words denoting new information. The most straightforward rationale for the speaker's behavior in this case refers to selfish rather than altruistic motives. Once new information has been introduced, the speaker can assume that a subsequent degradation in the acoustic signal for that information will not interfere with the listener's comprehension performance. The speaker can therefore afford to be more lazy in enunciation and, being selfishly inclined, take advantage of the situation. However, in doing so, the speaker is, in fact, creating a reliable correlation between stress and givenness, which could be exploited by the listener. This analysis leads to the counterintuitive, but confirmed, prediction that degraded information could signal to the listener that a word should have a coreferential antecedent, and hence enable a more efficient search for that antecedent than could have been triggered by nondegraded information. Thus, the speaker's behavior in this situation helps the listener, but the speaker's motivation is more selfish than cooperative.

It is quite possible that situations are rare in which the speaker must actually consider the listener's perspective in order to create linguistic patterns that aid the listener. In particular, the organization and use of linguistic knowledge might be quite similar when one is producing and comprehending speech (Levelt, 1989), and therefore, factors that ease or hinder language production may have similar effects on language comprehension. When language production processes are adjusted to help the speaker, the listener may be inadvertently aided. The result is a highly efficient communication system, but the efficiency may be created in a context of solipsism rather than, or in addition to, cooperation. The denominal verb phenomena discussed in this paper are, at present, quite consistent with these speculations, and they may provide one example of how pragmaticlooking effects can be obtained without pragmatics.

A second important question raised by this investigation is the theoretical status of the RD-ID distinction. In particular, what factors determine whether the members of a noun category will have RD or ID meanings when they are used as verbs? One possibility concerns the overwhelming saliency of a single feature in characterizing a noun category. For example, the single feature that unites all vehicles is that they are used for travel, and the single feature that unites all musical instruments is that they are used to make music. The dominance of such features suggests that they are almost definitional for these categories. For an object to be a musical instrument, it must possess the ability to make music. For an object to be a dwelling, 
it must have the capacity to house living things. Such features seem both necessary and sufficient for determining whether an object is a musical instrument or a dwelling. When nouns from these categories are used as verbs, these critical, definitional features stand a good chance of being incorporated into the verb meaning. In contrast, idiosyncratic categories do not seem to possess a definitional feature common to all members. Thus, body parts and tools serve a variety of rather distinct functions, and these idiosyncratic functions might be more salient than any function or feature potentially shared by all category members.

The prediction based on this account is that RD denominal verbs will be drawn from noun categories that fit the "classical" view of category structure (Smith \& Medin, 1981), in that membership in such categories depends on possessing a set of necessary and sufficient features. ID denominals, on the other hand, will be drawn from categories that have more of a "family resemblance" structure. One way to evaluate this hypothesis would be to present subjects with the name of a category or a list of category members and ask them to list what, if anything, the category members have in common. Based on some characteristic of the subjects' responses, such as the time needed to generate a shared trait, the categories could be divided into definitional and nondefinitional classes. Nouns from these categories could then be used in experiments similar to those described in this paper. One would expect that the denominal verbs from definitional categories would act in an RD fashion (e.g., relatively fast verb generations and similar verb meanings), whereas denominal verbs from nondefinitional categories would act in an ID fashion.

Another factor that could be relevant to denominal verb usage is the relative abstractness of a shared trait. For instance, one could claim that both animal and tool denominals are in some sense rule derived, because many animal verbs have the meaning "To act like an X" and many tool verbs mean "to use an X." However, the diversity of animal behaviors and tool uses is so great that both the speaker and the listener must specify the precise nature of these abstract "rule" meanings during denominal verb formation and interpretation. Hence, the idiosyncrasies in ID denominals might, in some cases, occur not at the level of the general meaning shared by the denominalized members of a category, but in the specific instantiations of the meaning. This need to assign a concrete meaning to an $a b-$ stract variable could hinder the production and comprehension of denominals in these and similar categories. In contrast, the precise manner in which one "paprikas" a goulash or "parsleys" a ragout seems less important to the core meaning "add X to food," and so the basic rule is sufficient for producing and interpreting a denominalized herb or spice. If correct, this account would predict that the more abstract the general meaning of denominal verbs in a category, the more difficult they should be to produce and comprehend.

Regardless of its eventual interpretation, the distinction between RD and ID denominal verbs has consistent, predictable effects on this form of lexical innovation. Thus, one implication of these results is programmatic. Psychologists have been concerned with highly skilled human performance since the beginning of the field. As with all skills learned to high degrees of fluency, language is used extensively for creative and æsthetic effects, which are appreciated by listeners and encouraged by society. Although long traditions of discussing poetry, drama, and other forms of language play exist in philosophy and linguistics, psychology has for the most part steered clear of these topics. The primary reason for this avoidance seems to be an apparent lack of constraint in such areas. If the only rule in the game is that no fast rules exist, gaining an explicit, rather than intuitive, understanding of the game could be a frustrating endeavor. Constraints on thought and behavior seem to be both a prerequisite for psychological investigation and the very object of study. If fruitful investigations of language creativity are to proceed, we must assume that constraints or biases predictably govern this type of language just as they govern the syntax and phonological form of ordinary language use. The reliability and robustness of the effects presented here suggest that psycholinguistic techniques can be used to study lexical innovation just as they can be used profitably to study such topics as lexical development in children and lexical access in adults. Indeed, studies of interactions between these areas are quite possible, and Clark (1982) has analyzed denominal verbs created by children, in terms of general issues in lexical development.

Finally, although in this paper I have focused on the advantages of RD verbs in comparison with ID verbs, it must be recognized that the bias against the latter class is probabilistic, since many ID verbs exist in English. How are we to account for the existence of ID denominal verbs? First, once a meaning for an ID verb becomes conventionalized, it can be entered into the lexicon and recalled for later use. Speakers and listeners would then not have to construct meanings for ID verbs, but would only have to recall them. Second, perhaps expertise in a particular domain can aid the production and comprehension of ID denominal verbs. Perhaps zoologists would be able to invent verb meanings for animal terms very quickly, and might even tend to choose such nouns for denominalization over nouns from RD categories. Third, the very ease with which $\mathrm{RD}$ verbs are understood might be a drawback in terms of appreciation. Perhaps denominal verbs are æsthetically more pleasing if their meanings are somewhat "translucent" rather than transparent or completely opaque. Tourangeau and Sternberg $(1981,1982)$ have found that metaphors are rated most pleasing if they are intermediate in difficulty, and a similar relationship between appreciation and interpretability may exist for denominal verbs.

\section{REFERENCES}

Battig, W. F., \& Montague, W. E. (1969). Category norms for verbal items in 56 categories: A replication of the Connecticut category norms. Journal of Experimental Psychology, 80, (3, Pt. 2).

BAUER, L. (1983). English word-formation. Cambridge: Cambridge University Press. 
ClARK, E. V. (1982). The young word maker: A case study of innovation in the child's lexicon. In E. Wanner \& L. Gleitman (Eds.), Language acquisition: The state of the art (pp. 390-425). New York: Cambridge University Press.

Clark, E. V. (1983). Meanings and concepts. In P. Mussen (Ed.), Manual of child psychology (Vol. 3, pp. 787-840). New York: Wiley.

Clark, E. V., \& Clark, H. H. (1979). When nouns surface as verbs. Language, 55, 767-811.

FowLER, C. A., \& Housum, J. (1987). Talkers signaling of "new" and "old" words in speech and listeners' perception and use of the distinction. Journal of Memory \& Language, 26, 489-504.

FRANCIS, W. N., \& KuČERA, H. (1982). Frequency analysis of English usage: Lexicon and grammar. Boston: Houghton-Mifflin.

GRICE, H. P. (1975). Logic and conversation. In P. Cole \& J. L. Morgan (Eds.), Syntax and semantics: Vol. 3. Speech acts (pp. 41-58). New York: Academic Press.

KELLY, M. H. (1988). Phonological biases in grammatical category shifts. Journal of Memory \& Language, 27, 343-358.

KELLY, M. H. (1992). Using sound to solve syntactic problems: The role of phonology in grammatical category assignments. Psychological Review, 99, 349-364.

LeVelt, W. J. M. (1989). Speaking: From intention to articulation. Cambridge, MA: MIT Press.

Mervis, C. B., Catlin, J., \& Rosch, E. (1976). Relationships among goodness-of-example, category norms, and word frequency. Bulletin of the Psychonomic Society, 7, 283-284.

SHERMAN, D. (1975). Noun-verb stress alternation: An example of lexical diffusion of sound change. Linguistics, 159, 43-71.

Smith, E. E., \& Medin, D. L. (1981). Categories and concepts. Cambridge, MA: Harvard University Press.

TOURANGEAU, R., \& SternberG, R. J. (1981). Aptness in metaphor. Cognitive Psychology, 13, 27-55.

Tourangeau, R., \& STERnBERG, R. J. (1982). Understanding and appreciating metaphors. Cognition, 11, 203-244.

Webster's ninth new collegiate dictionary. (1986). Springfield, MA: Merriam-Webster.

\section{NOTES}

1. Although each subject received 72 sentence pairs, only 36 noun pairs were used, with each noun appearing in two judgments. In order to reduce idiosyncrasies related to the particular sentences selected for the experiment, the results for each noun pair were collapsed over the sentences in which they appeared. Hence, the degrees of freedom for the items analysis is 35 rather than 71 .

2. Screening particular uses of $\mathrm{RD}$ denominals for whether they in fact followed the rule for their category was judged unacceptable. This paper claims that $\mathrm{RD}$ terms will be understood better than ID terms not only because they can follow a predictable meaning rule, but because they regularly do follow such rules when used by speakers. A fair test of this claim requires an unbiased sample of $\mathrm{RD}$ sentences, and hence the possibility that some rule violations will appear in the sample. The fact that predicted results were obtained despite the presence of deviant sentences increases confidence in the relevance of the rule/idiosyncratic distinction to denominal verb formation.

3. Two other reasons for the small effects in Experiment 4 could be the inclusion of function words in the calculations, which have relatively little semantic value, and the classification of nouns differing in number and verbs differing in tense into different word type categories. A re-analysis was therefore performed that excluded function words and classified noun pairs like cat-cats and verb pairs like play-played tokens of the same type. The new analysis produced slightly stronger results than did the original analysis. The type/token ratio of paraphrases of RD verbs was .22, whereas the ratio for paraphrases of ID verbs was $.27[t(70)=$ $2.48, p<.02$ ].

\section{APPENDIX A}

Item Pairs Used in Experiments, Listed by Rule-Derived Categories

\begin{tabular}{ll} 
Vehicle & Color \\
scooter-skillet & violet-spatula \\
elevator-alligator & turquoise-hamster \\
tractor-cellar & scarlet-attic \\
cab-rug & chartreuse-dinette \\
streetcar-backbone & orange-ankle \\
tricycle-screwdriver & beige-awl \\
Musical Instrument & Flavoring \\
saxophone-casserole & parsley-oven \\
bassoon-raccoon & paprika-iguana \\
horn-porch & horseradish-balcony \\
banjo-footstool & garlic-hammock \\
harmonica-esophagus & cinnamon-skeleton \\
tuba-anvil & nutmeg-yardstick \\
Dance & Dwelling \\
cancan-griddle & bungalow-colander \\
flamenco-flamingo & dorm-deer \\
ballet-facade & cottage-bathroom \\
chacha-sofa & igloo-bookcase \\
Charleston-abdomen & tepee-neuron \\
softshoe-sawhorse & mansion-hatchet \\
\hline
\end{tabular}


APPENDIX B

Sample Sentence Pairs Used in Experiment 3

Verb Condition

1RD: She chartreused the sheets.

1ID: She dinetted the living room.

2RD: The tea was cinnamoned before it was served.

2ID: The killer skeletoned his victim; flesh was everywhere.

3RD: We elevatored up to the fifth floor.

3ID: Alligatoring along, it took her a very long time to complete her walk.

4RD: She made her pizza spicy because she paprikaed it.

4ID: Whenever he gets scared, he iguanas.

5RD: The eskimos igloo to keep warm.

5ID: I bookcased the wall.

6RD: I cottaged by the ocean.

6ID: I bathroomed my toothbrush and toothpaste.

7RD: I dormed all freshman year.

7ID: He deered the part.

8RD: The band tubaed.

8ID: He anviled the rod.

No-Verb Condition

1RD: The hillbilly played the banjo.

1ID: The cat rested on the footstool.

2RD: Paprika is orange.

2ID: Iguanas are cold-blooded.

3RD: My uncle has a tepee in his backyard.

3ID: A neuron is a nerve cell.

4RD: The tuba section is one of the wildest in the band.

4ID: The Anvil Club is the dining hall for the Wharton MBAs.

5RD: Cabs are expensive in New York City.

5ID: I need to vacuum my rug.

6RD: They danced the Charleston.

6ID: She had a protruding abdomen.

7RD: I hate the smell of garlic.

7ID: I have seen hammocks in people's gardens.

8RD: Is there parsley in the tuna fish?

8ID: Put the bread in the oven.

Note-ZRD, rule derived; ID, idiosyncratically derived.

(Manuscript received July 12, 1996; revision accepted for publication February 12, 1997.) 